\title{
SOLUBILITY OF DEUTERIUM IN YSZ10 ZIRCONIUM DIOXIDE NANOPARTICLES
}

\author{
V. B. Vykhodets, T. E. Kurennykh* \\ M.N. Miheev Institute of Metal Physics, Ural Branch of the Russian Academy of Sciences, \\ 18 S. Kovalevskoy St., 620990, Ekaterinburg, Russian Federation \\ *Corresponding author. E-mail: kurennykh@imp.uran.ru \\ Address for correspondence: 18 S. Kovalevskoy St., 620990, Ekaterinburg, Russian Federation \\ Tel.: +7 34337838 46; fax: +7 (343) 3745244
}

For cubic zirconia doped with yttrium oxide, data have been obtained that attest to the high sensitivity of the solubility of deuterium in nanopowders to oxygen deficiency in the volume of nanoparticles and to surface structural defects. The investigation is performed on nanopowders synthesized by laser sputtering of a ceramic target. An accelerating technique of nuclear reactions is used to measure deuterium concentration. It has also been found that the deuterium concentration on the surface of nanoparticles decreases when they are irradiated with deuterons, this effect being due to the selective sputtering of dissolved deuterium atoms by accelerator beam ions. It is concluded that investigations of deuterium solubility and ion sputtering of deuterium atoms by accelerated deuterons are an effective tool for obtaining information on the defect structure of oxide nanoparticles.

Keywords: solubility of deuterium, nanopowder, nanoparticle, oxygen deficiency, nuclear reaction technique, ion sputtering, zirconia

DOI: $10.17804 / 2410-9908.2017 .5 .006-012$

\section{References}

1. Kamat P. V. $\mathrm{TiO}_{2}$ Nanostructures: Recent Physical Chemistry Advances // J. Phys. Chem. C. - 2012. - Vol. 116. - P. 11849-11851. - DOI: 10.1021/jp305026h

2. Size-Controlled Growth of $\mathrm{TiO}_{2}$ nanowires by Oxidation of Titanium Substrates in the Presence of Ethanol Vapor / S. Daothong, N. Songmee, S. Thongtem, P. Singjai // Scr. Mater. - 2007. Vol. 57. - P. 567-570. - DOI: 10.1016/j.scriptamat.2007.06.030

3. High-Temperature Ferromagnetism in Co-implanted $\mathrm{TiO}_{2}$ Rutile / N. Akdogan, A. Nefedov, H. Zabel, K. Westerholt, H.-W. Becker, C. Somsen, Ş. Gök, A. Bashir, R. Khaibullin, L. Tagirov // J. Phys. D: Appl. Phys. - 2009. - Vol. 42. - P. 115005-1-115005-11. DOI: $10.1088 / 0022-3727 / 42 / 11 / 115005$

4. Direct Observation of Tunable Surface Structure and Reactivity in $\mathrm{TiO} 2$ Nanopowders / V. B. Vykhodets, K. G. Johnson, T. E. Kurennykh, I. V. Beketov, O. M. Samatov, A. I. Medvedev, E. A. A. Jarvis // Surface Science. - 2017. - Vol. 665 C. - P. 10-19. - DOI: 10.1016/j.susc.2017.08.001

5. Brunauer S., Emmett P. H., Teller E. Adsorption of gases in multimolecular layers // J. Am. Chem. Soc. - 1938. - Vol. 60. - P. 309-319. - DOI: 10.1021/ja01269a023

6. Sputtering by Particle Bombardment I. Physical sputtering of Single-Element Solid / ed. by R. Behrisch. - Berlin, Heidelberg, New York : Springer-Verlag, 1981. - 335 p.

7. Sputtering by Particle Bombardment II. Sputtering of Alloys and Compounds, Electron and Neutron Sputtering. Surface Topography / ed. by R. Behrisch. - Berlin, Heidelberg, New York, Tokyo : Springer-Verlag, 1983. - 485 p. 
Подана в журнал: 06.10.2017

УДК 544.6:544.16:539.2:517.958

DOI: $10.17804 / 2410-9908.2017 .5 .006-012$

\title{
РАСТВОРИМОСТЬ ДЕЙТЕРИЯ В НАНОЧАСТИЦАХ ДИОКСИДА ЦИРКОНИЯ YSZ10
}

\author{
В. Б. Выходец, Т. Е. Куренных \\ Федеральное государственное бюджетное учреждение науки \\ Институт физики металлов им. М.Н. Михеева Уральского отделения Российской академии наук, \\ ул. С. Ковалевской, 18, Екатеринбург, Российская Федерации \\ *Ответственный автор. Электронная почта: kurennykh@imp.uran.ru \\ Адрес для переписки: ул. С. Ковалевской, 18, 620990, Екатеринбург, Российская Федерация \\ Телефон: +7 (343)3783846; факс: +7 (343)3745244
}

Для кубического диоксида циркония, легированного оксидом иттрия, получены данные, свидетельствующие о высокой чувствительности растворимости дейтерия в нанопорошках к кислородному дефициту в объеме наночастиц и к поверхностным структурным дефектам. Исследование выполнено на нанопорошках, синтезированных по технологии лазерного распыления керамической мишени. Для измерения концентрации дейтерия использована ускорительная методика ядерных реакций. Также установлено, что концентрация дейтерия на поверхности наночастиц уменьшается при их облучении дейтронами, этот эффект обусловлен селективным распылением растворенных атомов дейтерия ионами пучка ускорителя. Сделано заключение, что исследования растворимости дейтерия и ионного распыления атомов дейтерия ускоренными дейтронами являются эффективным инструментом для получения информации о дефектной структуре оксидных наночастиц.

Ключевые слова: растворимость дейтерия, нанопорошок, наночастица, кислородный дефицичт, методика ядерных реакиий, ионное распыление, диоксид ичиркония.

\section{1. Введение}

Оксидные нанопорошки широко используются в различных областях техники: в машиностроении - для получения конструкционных материалов, электронике, оптоэлектронике, катализе, электрохимических генераторах, преобразователях солнечной энергии, устройствах для получения водорода, спинтронике, газовых сенсорах и т. д. [1-3]. В связи с этим во многих работах проводились исследования кристаллической и дефектной структуры оксидных наночастиц, их магнитных свойств, эволюции дефектной структуры при термической обработке нанопорошков и т. д. Соответствующие исследования проводились с использованием широкого набора методик: рентгеновской дифракции, просвечивающей электронной микроскопии высокого разрешения, позитронной аннигиляции, спектроскопии Допплеровского уширения, намагниченности насыщения. В этих исследованиях были получены данные о существовании в наночастицах оксидов широкого спектра точечных дефектов: моновакансий кислорода и металла, кластеров кислородных вакансий, междоузельных атомов металла и более сложных по составу точечных дефектов. Общей проблемой подобного типа исследований является получение информации о концентрации точечных дефектов в наночастицах, включая данные о концентрациях точечных дефектов, расположенных на поверхности и в объеме наночастиц, типах и характеристиках дефектов. В целом с помощью существующих классических методик не удалось получить детальную информацию о дефектной структуре оксидных наночастиц и актуальной задачей является разработка новых подходов для исследования этих вопросов. 
В настоящей работе ставилась задача получения информации о дефектной структуре оксидных наночастиц с помощью измерений в них растворимости дейтерия. Эти исследования были начаты в работе [4], их постановка определялась следующими соображениями. В массивных оксидах металлов с совершенной структурой водород практически не растворим, и заметная растворимость имеет место только в оксидах, содержащих структурные вакансии в кислородной подрешетке, следовательно, можно ожидать, что в оксидных наночастицах растворимость водорода будет чувствительна к точечным дефектам, расположенным в объеме н на поверхности наночастиц, а также к их типу. Поскольку атомы водорода (дейтерия) очень быстро диффундируют в твердых телах, в случае малого размера частиц можно определенно рассчитывать на то, что атомы водорода (дейтерия) будут взаимодействовать с точечными дефектами, расположенными как в объеме, так и на поверхности наночастиц. Другими словами, подход к исследованию точечных дефектов в оксидных наночастицах, основанный на измерении растворимости водорода, может иметь универсальный характер с точки зрения пространственного расположения дефектов. Выбор для настоящего исследования газообразного дейтерия, а не водорода в качестве среды, в которой проводился отжиг нанопорошков, обусловлен двумя обстоятельствами. Во-первых, концентрация дейтерия в нанопорошках легко может быть измерена с помощью ускорительной методики ядерных реакций. В противоположность этому, измерение концентрации основного изотопа водорода (протия) в нанопорошках является более сложной задачей. Во-вторых, использование атомов дейтерия в качестве зонда предпочтительнее, чем атомов протия, поскольку дейтерий практически отсутствует в природной смеси изотопов водорода, что позволяет избежать его неконтролируемого поступления в порошки.

В настоящее время нет возможности сделать обоснованные прогнозы по поводу эффективности предлагаемого подхода с точки зрения дифференциации типов точечных дефектов, например, нельзя утверждать, что методика, основанная на измерении растворимости дейтерия, будет способна отличить моновакансии от кластеров вакансий или вакансии в катионной и анионной подсистемах оксида. Учитывая это, мы рассматриваем исследуемый здесь подход как дополнение к существующим методикам, при использовании которых может быть идентифицирован тип точечных дефектов.

Выбор в работе в качестве объекта исследований кубического диоксида циркония, легированного оксидом иттрия, обусловлен тем, что в этом массивном оксиде имеются точечные дефекты, а именно - вакансии в кислородной подрешетке и связанный с ними кислородный дефицит. По этой причине в наночастицах легированного диоксида циркония неизбежно будут присутствовать точечные дефекты как на поверхности, так и в объеме наночастиц. При выборе материала для исследования также принималось во внимание что диоксид YSZ10, содержащий 10 мол. \% оксида $\mathrm{Y}_{2} \mathrm{O}_{3}$, является важным техническим материалом.

\section{2. Образцы и методики}

Исследование проведено на нанопорошках, полученных с помощью технологии лазерного испарения керамической мишени. Исходным материалом для изготовления мишени был коммерческий микропорошок YSZ10 $\left(\mathrm{ZrO}_{2}-10\right.$ моль \% $\left.\mathrm{Y}_{2} \mathrm{O}_{3}\right)$. По данным рентгенофазового анализа (РФА), он имел кубическую структуру с периодом решётки 0,5145 нм. Формула оксида может быть представлена в виде $\mathrm{Zr}_{0,82} \mathrm{Y}_{0,18} \mathrm{O}_{2-\delta}$, или $\mathrm{Zr}_{0,82} \mathrm{Y}_{0,18} \mathrm{O}_{1,91}$. Из соображений электронейтральности можно сделать следующую оценку концентрации структурных кислородных вакансий в оксиде YSZ10: она равна $\delta / 3=3$ ат. \%. Перед изготовлением мишени кислородная подсистема исходного микропорошка была обогащена изотопом ${ }^{18} \mathrm{O}$. Для этого проводился отжиг исходного порошка в проточном кислороде, обогащённом на $80 \%$ изотопом ${ }^{18} \mathrm{O}$. Температура отжига была $800{ }^{\circ} \mathrm{C}$, продолжительность 24 ч, давление кислорода 1 атм. Отжиг проводился в алундовой трубе, в ней размещались таблетки, полученные прессованием из исходного порошка. Диаметр 
таблеток был 15 мм, толщина 10-15 мм, плотность - 35-40 \% от теоретической. Прессование проводилось при комнатной температуре. Плотность таблеток составляла 35-40 \% от теоретической. После отжига проводился размол таблеток в механической ступке из оксида циркония. Удельная поверхность порошка после размола таблеток составила $2,2 \mathrm{~m}^{2} /$ г. По данным методики ядерного анализа (NRA), степень обогащения кислородной подсистемы оксида изотопом ${ }^{18} \mathrm{O}$ была около $15 \%$. Из микропорошка, обогащённого изотопом ${ }^{18} \mathrm{O}$, была изготовлена таблетка диаметром 60 мм, которая служила мишенью при использовании технологии лазерного испарения. При изготовлении мишени осуществлялось прессование порошка на воздухе при комнатной температуре.

Для получения нанопорошков использовался волоконный иттербиевый лазер с длиной волны 1,07 $\mu$ м, максимальная мощность 1 кВт. Для того чтобы исключить протекание процессов кислородного изотопного обмена при синтезе нанопорошков, лазерное испарение керамической мишени проводилось в среде инертных газов (аргона или гелия). Методика, которая использовалась в работе, предполагает синтез нанопорошков с различным средним размером частиц в широком диапазоне значений удельной поверхности. С этой целью при синтезе варьировались сорт инертного газа (аргон или гелий), давление газа, мощность лазера, использовался как импульсный, так и непрерывный режим лазерного облучения мишени. При использовании импульсного режима длительность импульсов была $60 \mu$ с, частота 5 кГц. Излучение лазера фокусировалось на мишени с помощью оптической системы Optoskand d25 f60/200, она обеспечивала плотность мощности лазерного облучения на поверхности мишени до $10^{7} \mathrm{BT} / \mathrm{cm}^{2}$. Для достижения равномерного износа мишени при лазерном облучении осуществлялось вращение мишени вокруг своей оси и её возвратно-поступательные перемещения в плоскости, перпендикулярной оси лазерного пучка.

Удельные поверхности порошков определялись с помощью БЭТ-методики [5]. По данным просвечивающей электронной микроскопии, нанопорошки имели узкие распределения частиц по размерам и частицы с размером больше 1 мкм в них отсутствовали. Это связано с тем, что установка для синтеза содержала устройство для отделения крупных частиц. Все нанопорошки и исходный микропорошок были однофазными и имели кубическую решётку. Ширина дифракционных линий монотонно увеличивалась при возрастании удельной поверхности порошков. Форма линий свидетельствовала о практическом отсутствии в нанопорошках аморфной фазы и микронапряжений.

Средние концентрации дейтерия ${ }^{2} \mathrm{H}$ в порошках определяли с помощью методики ядерных реакций на 2 MB ускорителе Ван де Граафа, использовали реакцию ${ }^{2} \mathrm{H}(\mathrm{d}, \mathrm{p})^{3} \mathrm{H}$ при энергии дейтронов $650 \mathrm{kэB}$. Для проведения измерений частицы нанопорошков оксида впрессовывали в индиевую пластину. Измерения в основном проводили при комнатной температуре образцов, а некоторые исследования - при 60 и $-60{ }^{\circ} \mathrm{C}$. В этих случаях применяли специальный держатель образцов. В нем для получения необходимой температуры использовали проточный жидкий азот и резистивный нагреватель. Температуру образца измеряли с помощью хромель-алюмелевой термопары, диаметр первичного пучка дейтронов составлял 2 мм. Регистрация продуктов ядерной реакции (протонов) проводилась с помощью кремниевого поверхностно-барьерного детектора диаметром около 10 мм, угол регистрации был $160^{\circ}$. Доза облучения, т. е. число частиц первичных пучков, падающих на образец, определялась с помощью вторичного монитора, статистическая погрешность в этих измерениях была около $0,5 \%$. При математической обработке спектров продуктов ядерной реакции использовали приближенную процедуру сравнения спектров от исследуемых образцов и эталонного образца с постоянной по глубине концентрацией дейтерия. Систематическая ошибка в измерении концентрации, связанная с использованием приближенной процедуры, не превышала $1 \%$. Эталонным образцом при измерении концентрации дейтерия был дейтерид $\mathrm{ZrCr}_{2} \mathrm{D}_{4}$. Среднеквадратичные ошибки при измерениях концентрации дейтерия были около $10 \%$. 
Изотермические отжиги нанопорошков в газообразном дейтерии проводили в кварцевой трубе при $400{ }^{\circ} \mathrm{C}$, продолжительность отжигов была 1 ч, давление дейтерия - 0,6 атм., Кварцевая труба с порошками размещалась внутри массивного металлического цилиндра. В этих условиях градиент температуры в зоне расположения порошков практически отсутствовал. Температуру измеряли с помощью хромель-алюмелевой термопары с точностью 1 ${ }^{\circ} \mathrm{C}$. Время, необходимое для достижения температуры отжига, составляло 10 мин, а время охлаждения образцов до комнатной температуры - 1 мин. Удельная поверхность порошков при отжигах уменьшалась, максимальное ее изменение составляло 1,5 раза.

\section{3. Результаты и обсуждение}

Из данных рис. 1 видно, что концентрация дейтерия $C_{\mathrm{D}}$ линейно возрастала при увеличении удельной поверхности $S$ нанопорошков, при этом значение концентрации дейтерия при экстраполяции на $S=0$ составило $C_{\mathrm{D} 0} \approx 0,75$ ат. \%.

В работе [4] при исследовании нанопорошков диоксида титана было установлено, что концентрация дейтерия уменьшалась при облучении порошков дейтронами. Из рис. 2 видно, что в случае оксида YSZ10 также имеет место уменьшение концентрации $C_{\mathrm{D}}$, при этом $C_{\mathrm{D}}$ уменьшалась до значения, близкого к концентрации дейтерия в объеме наночастиц. Соответствующие исследования были проведены при комнатной температуре нанопорошка, а также при -60 и $60{ }^{\circ} \mathrm{C}$. Было установлено, что кинетика уменьшения концентрации дейтерия не зависела от температуры образца. В то же время, концентрация дейтерия в порошках не изменялась при их хранении на воздухе или в вакууме в течение нескольких суток при комнатной температуре.

Полученные результаты были интерпретированы в модели, согласно которой атомы дейтерия взаимодействуют с точечными дефектами, расположенными в объеме оксида YSZ10, а именно - со структурными кислородными вакансиями, а также с точечными дефектами, локализованными вблизи поверхности наночастицы. Об этом свидетельствует наличие линейной зависимости $C_{\mathrm{D}}(S)$. Обращает на себя внимание, что концентрация дейтерия в объеме наночастиц $C_{\mathrm{D} 0}$ имеет тот же порядок величины, что и концентрация структурных кислородных вакансий в оксиде YSZ10. В разд. 2 приведено ее значение - 3 ат. \%, что свидетельствует о том, что энергия связи атома дейтерия с кислородной вакансией не очень сильно отличается от энергии связи атомов дейтерия в молекуле $\mathrm{D}_{2}$, т. е. превышает 2 эВ.

На основании полученных результатов было сделано заключение, что уменьшение концентрации дейтерия в нанопорошках при их облучении дейтронами обусловлено распылением ионами пучка атомов дейтерия, расположенных вблизи поверхности наночастиц. Распыление твердых тел при ионной бомбардировке было предметом многочисленных исследований $[6,7]$, они представляют большой интерес для технических приложений и для получения информации об энергетических состояниях атомов на поверхности твердых тел. В то же время, по нашим данным, в литературе отсутствуют сведения о селективном распылении ионами пучка атомов примесей, локализованных вблизи поверхности наночастиц. Вероятно, полученные в работе данные по этому эффекту свидетельствуют о слабом взаимодействии атомов дейтерия с точечными дефектами на поверхности наночастиц. В контексте данной работы нужно отметить, что исследование этого эффекта на оксидных нанопорошках позволит получить новые данные не только по эффекту распыления, но также по дефектной структуре поверхностного слоя оксидных наночастиц и состоянию атомов водорода в этом слое. 


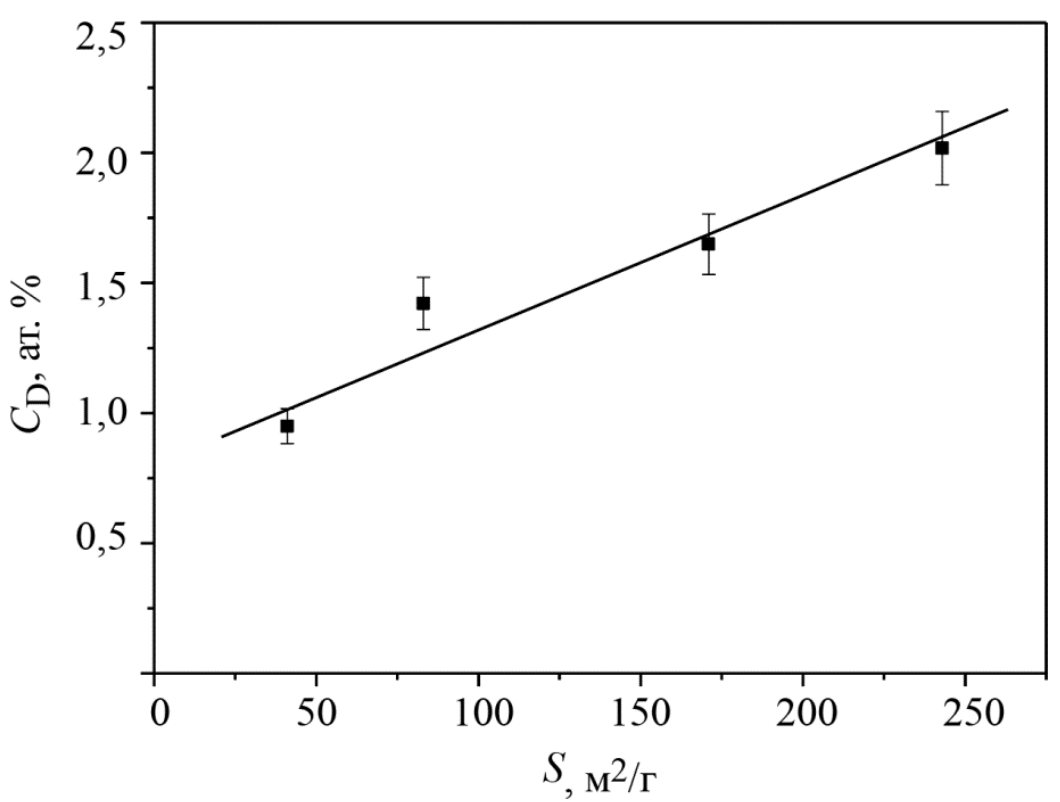

Рис. 1. Зависимость концентрации дейтерия $C_{\mathrm{D}}$ в нанопорошках оксида YSZ10 от удельной поверхности $S$

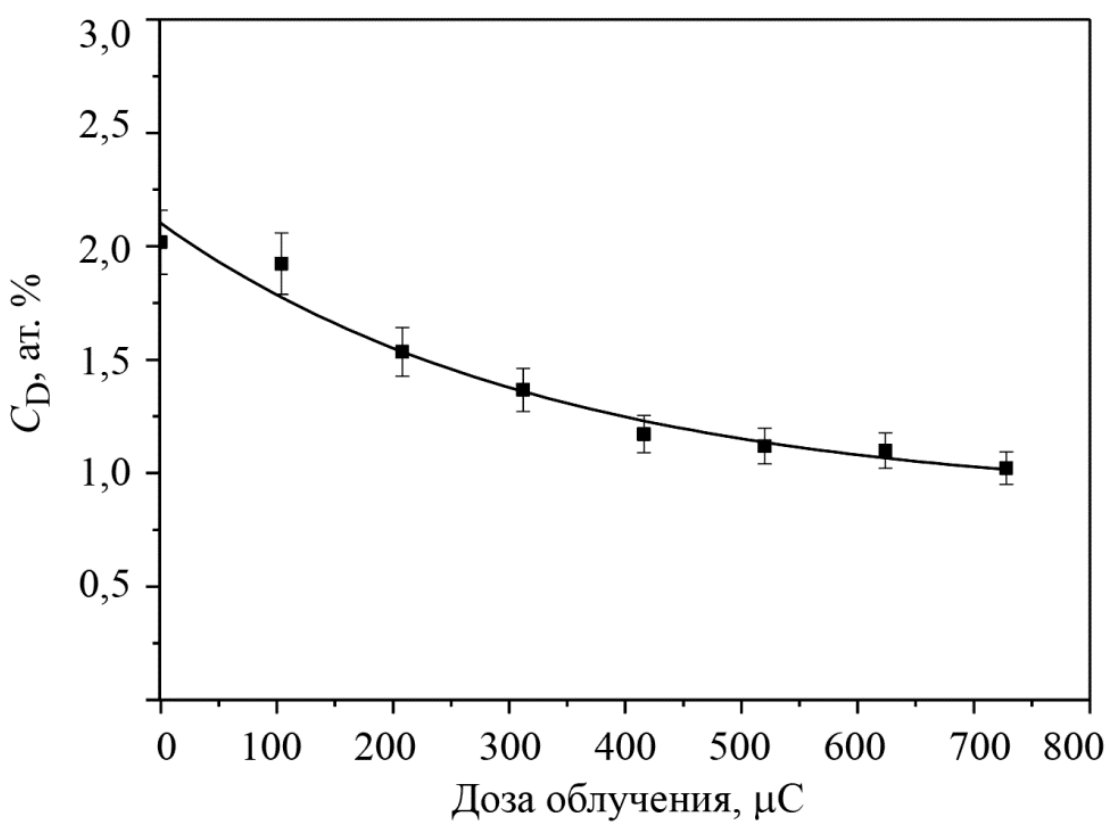

Рис. 2. Зависимость концентрации дейтерия $C_{\mathrm{D}}$ от дозы облучения дейтронами для нанопорошка YSZ10 с удельной поверхностью $S=247 \mathrm{~m}^{2} / \Gamma$

\section{4. Заключение}

1. Установлено, что растворимость дейтерия в оксидных нанопорошках чувствительна к точечным дефектам, расположенным в объеме и на поверхности оксидных наночастиц.

2. Концентрация дейтерия на поверхности оксидных наночастиц уменьшается при их облучении дейтронами за счет эффекта распыления.

3. Результаты работы могут быть использованы для получения информации о дефектной структуре оксидных наночастиц. 


\section{Благодарность}

Работа выполнена в рамках проекта УрО РАН №15-17-2-10.

\section{Литература}

1. Kamat P.V. $\mathrm{TiO}_{2}$ Nanostructures: Recent Physical Chemistry Advances. J. Phys. Chem. C, 2012, vol. 116, pp. 1149-11851. DOI: 10.1021/jp305026h

2. Daothong, S., Songmee, N., Thongtem, S., Singjai, P. Size-Controlled Growth of $\mathrm{TiO}_{2}$ nanowires by Oxidation of Titanium Substrates in the Presence of Ethanol Vapor. Scr. Mater., 2007, vol. 57, pp. 567-570. DOI: 10.1016/j.scriptamat.2007.06.030

3. Akdogan N., Nefedov A., Zabel H., Westerholt K., Becker H.-W., Somsen C., Gök Ş., Bashir A., Khaibullin R., Tagirov L. High-Temperature Ferromagnetism in Co-implanted $\mathrm{TiO}_{2}$ Rutile. J. Phys. D: Appl. Phys., 2009, vol. 42, pp. 115005-1-115005-11. DOI: 10.1088/00223727/42/11/115005

4. $\quad$ Vykhodets V.B., Johnson K.G., Kurennykh T.E., Beketov I.V., Samatov O.M., Medvedev A.I., Jarvis E.A.A. Direct Observation of Tunable Surface Structure and Reactivity in TiO2 Nanopowders. Surface Science, 2017, vol. 665 C, pp. 10-19. DOI: 10.1016/j.susc.2017.08.001

5. Brunauer S., Emmett P.H., Teller E. Adsorption of gases in multimolecular layers. J. Am. Chem. Soc., 1938, vol. 60, pp. 309-319. DOI: 10.1021/ja01269a023

6. Sputtering by Particle Bombardment I: Physical sputtering of Single-Element Solid, R. Behrisch, ed., Berlin, Heidelberg, New York, Springer-Verlag, 1981, 335 p.

7. Sputtering by Particle Bombardment II: Sputtering of Alloys and Compounds, Electron and Neutron Sputtering. Surface Topography, R. Behrisch, ed., Berlin, Heidelberg, New York, Tokyo, Springer-Verlag, 1983, 485 p. 